genügt ein mehrstündiges ruhiges Stehenlassen bei gewöhnlicher Temperatur im verschlossenen Glase mit Glasstopfen vollständig zur Zersetzung des Ferrisulfats, während beim Erwärmen der Mischung des Liquors mit Jodkalium ohne Salzsäure sich basisches Sulfat oft früher abscheidet, bevor die Einwirkung des Jodkaliums beendet ist. Dadurch wird das Resultat der Analyse falsch, weil Jodkalium auf gefälltes basisches Ferrisulfat nicht mehr einwirkt.

1) Ein völlig normales Präparat p. sp. 1,4285 b. $15^{\circ} \mathrm{C}$.

a) 1,070 g. gaben mit $\mathrm{Na}^{2} \mathrm{~S}^{2} \mathrm{O}^{3}$ und $J$ $10,00 \% \mathrm{Fe}$.

b) 1,070 - - $-\mathrm{KJ}$ und $\mathrm{HCl}$ 10,04 -

c) 1,070 - - KJ ohne HCl 9,88 -

d) 1,070 - $\quad$ - 0,1892 g. $\mathrm{Fe}^{2} \mathrm{O}^{3}$ entsprechend 10,09 -

e) 1,070 - $\quad 25,88 \%$ Schwefelsäure $\mathrm{SO}^{4}$ - 9,86 im Mittel daher 10,03\% Eisen, davon 0,15\% Eisen als Oxyd, entsprechend $0,21 \% \mathrm{Fe}^{2} \mathrm{O}^{3}$. Die gefundene Schwefelsäure entspricht dem als Sulfat vorhandenem Eisen.

2) Ein stark saures, sonst reines Präparat p. sp. 1, 3160.
a) 1,4956 g. gaben mit $\mathrm{Na}^{2} \mathrm{~S}^{2} \mathrm{O}^{3}$ und $\mathrm{J}$
$5,78 \%$ Fe.
b) 1,4956
- $\mathrm{KJ}$ ohne $\mathrm{HCl}$
$5,70-$
c) $1,4956-\quad-\quad 0,3494 \mathrm{~g}$.
$23,36-\mathrm{SO}^{4}$

In Mittel daher $5,74 \% \mathrm{Fe}$. Diese verlangen $12,87 \% \mathrm{SO}^{4}$, daher $10,5 \%$ SO4 im Ueberschuss.

Ein völlig verfehltes Präparat!

Diese Methode zur Bestimmung des Oxychlorids und des basischen Ferrisulfats in den beiden hier in Rede stehenden Eisenpräparaten genügt daher vollständig den Eingangs dieser Arbeit angezogenen Forderungen der präcisen Charakterisirung eines pharmaceutischen Präparats, eignet sich aber auch vortrefflich zur Analyse der Oxychloride, basischen Sulfate und Nitrate des Eisens innerhalb gewisser Grenzen, denn zur Bestimmung des Oxychlorids des in jeder Beziehung sich eigenartig verhaltenden Liquor Ferri oxychlorati ist sie nicht zu gebrauchen.

\title{
3. Liquor Plumbi subacetici.
}

Die Vorarbeiten zum Liquor Plumbi subacetici für die gegenwärtige deutsche Pharmakopöe bezogen sich auf die Darstellung, den Einfluss kohlensäurehaltigen Bleioxyds auf dieselbe und endlich auf die Zusammensetzung des basischen Bleiacetats im Liquor. 
Die vortreffliche, schon vor Jahren von Hager empfohlene Methode zur Darstellung des Präparats bedurfte keiner Aenderung. Die abweichenden Gewichtsverhältnisse der Pharmacopoea borussica militaris, sowie die aus einander gehenden Ansichten, ob das Präparat $1 / 2,2 / 3$ oder $1 / 3$ Bleiacetat enthalte oder nur eine, in ihrem Gehalt wechselnde Lösung von Bleioxyd in gelöstem normalen Bleiacetat sei, bedurfte der Aufklärung und diese konnte nur auf experimentellem Wege gewonnen werden.

Die Analyse einer Anzahl Präparate wurde in nachstehender Weise ausgeführt.

Eine gewogene Menge des Liquor Plumbi subacetici wurde mit normaler Natriumcarbonatlösung gefällt, der Niederschlag gut ausgewaschen und nach dem Trocknen und Glühen gewogen. Das Filtrat wurde mit normaler Salzsäure übersättigt, durch Erhitzen die Kohlensäure beseitigt und der Ueberschuss der Säure mit NormalKali zurückgemessen.

1) Präparat mit ungeglühtem Bleioxyd, welches $9,9 \% 2 \mathrm{PbCO}^{3}$. $\mathrm{Pb}(\mathrm{OH})^{2}$ enthielt, in den Verhältnissen der Vorschrift der Pharmakopöe dargestellt. 60 g. Bleiacetat, 20 g. Bleioxyd, $200 \mathrm{~g}$. Wasser. Specifisches Gewicht des unmittelbar erhaltenen Filtrats 1,2422.

a) Der bei $110^{\circ}$ getrocknete, fast ganz weisse, unlöslicho Rückstand von der Bereitung des Präparats wog 2,0225 g., nach dem Glühen $1,8235 \mathrm{~g}$. Dies entspricht $0,72 \%$ und $0,65 \%$ der gesammten Menge des Präparats, oder auf $20 \mathrm{~g}$. des angewandten Lithargyrums berechnet $10,11 \%$ und 9,11\% desselben, also nahezu dem Gehalt des Bleioxyds an basischem Bleicarbonat. Da beim Glühen gar keine Schwärzung oder Reduction stattfand, so können nur minimale Mengen Essigsäure in diesem unlöslichen Rückstande vorhanden gewesen sein, entgegen der Annahme, dass dieser Rückstand wesentlich aus $1 / 6$ essigsaurem Blei bestehe, dessen Existenz mir überhaupt wenig wahrscheinlich erscheint.

b) $9,8115 \mathrm{~g}$. des Liquors gaben 1,9045 g. $\mathrm{PbO}=19,41 \% \mathrm{PbO}$ $=18,01 \% \mathrm{~Pb}$.

c) Zur Sättigung der Essigsäure wurden 11,8 C.C. Normalnatron verbraucht $=7,09 \% \quad \mathrm{C}^{2} \mathrm{H}^{3} \mathrm{O}^{2}$ (Essigsäuremolekel weniger 1 Atom Wasserstoff). 
Das Verhältniss des Bleis zur Essigsäure ist wie 1 Atom Blei: 1,38 Molekel Essigsäure, daher 3 Atome Blei auf 4 Molekel Essigsäure $={ }^{2} / 3$ Bleiacetat, $=\mathrm{Pb}^{3}\left\{\begin{array}{l}\left(\mathrm{C}^{2} \mathrm{H}^{3} \mathrm{O}^{2}\right)^{4} \\ (\mathrm{OH})^{2}\end{array}\right.$

Wenn das Atom Blei vierwerthig angenommen wird, wozu man nach seiner Verbindung mit Aethyl $\mathrm{Pb}\left(\mathrm{C}^{2} \mathrm{H}^{5}\right)^{4}$ etc. berechtigt ist, so springt die Analogie mit dem $2 / 3$ Eisen- und Aluminiumacetat in die Augen.

2) 60 g. Bleiacetat, 20 g. geglühte Bleiglätte und 200 C. C. Wasser.

a) Der bei $110^{\circ}$ getrocknete Rückstand wog 0,3305 g. $=0,11 \%$ des ganzen Präparats und 1,65\% der angewandten Bleiglätte; nach dem Glühen $0,2985 \mathrm{~g} .=0,10 \%$ des Präparats und $1,48 \%$ der Bleiglätte. Hier liegt der Erfolg des vorherigen Erhitzens des 9,95\% Bleisubcarbonat enthaltenden Bleioxyds auf der Hand. Dem entsprach auch das specifische Gewicht des unmittelbar erhaltenen Filtrats 1,2452.

b) 9,2545 des Liquors gaben $1,793 \mathrm{~g}$. $\mathrm{PbO}=17,98 \% \mathrm{~Pb}$.

c) Zur Sättigung der Eissigsäure wurden verbraucht 11,6 C.C. Normalnatron $=7,39 \% \quad \mathrm{C}^{2} \mathrm{H}^{9} \mathrm{O}^{2}$.

1 Atom Blei: 1,44 Molekel Essigsäure, daher $3 \mathrm{~Pb}: 4 \mathrm{C}^{2} \mathrm{H}^{3} \mathrm{O}^{2}$. Es ist also auch hier $2 / 3$ Acetat vorhanden.

Das Gleiche war in einer Anzahl käuflicher Präparate der Fall. In einem Präparat war das Verhältniss von Blei zur Essigsäure wie $4: 5$, hier war neben $2 / 3$ basisches $1 / 2$ basisches Acetat vorhanden; in einem andern schlecht bereiteten Präparat war das Verhältniss wie $4 \mathrm{~Pb}: 6 \mathrm{C}^{2} \mathrm{H}^{3} \mathrm{O}^{2}$, diesem entsprechen gleiche Molekel normales und $2 / 3$ basisches Acetat.

Aus den vorstehenden Analysen geht zweifellos hervor, dass der correkt bereitete Liquor Plumbi subacetici nur $2 / 3$ Bleiacetat enthält. Da dieses gleichzeitig das in Wasser und Alkohol löslichste basische Bleiacetat und seiner Zusammensetzung nach constant ist, so lag gar kein Bedürfniss vor, die Vorschrift der Pharmakopöe zu ändern.

Eine grössere Menge Bleiglätte auf dieselbe Menge Bleiacetat ist ohne Einfluss auf die Zusammensetzung des Präparats. Die Pharmacop. milit. boruss. liess nur $2 / 3$ der in der deutschen Pharmakopöe vorgeschriebenen Menge Bleiacetat auf dieselbe Menge Bleioxyd und weniger Wasser nehmen. 
Es wurde völlig normaler, nach der Militär-Parmakopöe bereiteter Bleiessig von p. sp. $\mathbf{1 , 2 5 5}$ in derselben Weise analysirt.

$11,6205 \mathrm{~g}$. gaben 2,396 g. $\mathrm{PbO}=19,14 \% \mathrm{~Pb}$ und erforderten 13,95 C.C. normaler Natriumcarbonatlösung zur Bindung der Essigsäure; dies entspricht 0,8231 g. $\mathrm{C}^{8} \mathrm{H}^{3} \mathrm{O}^{2}=7,08 \%$.

Es verhält sich Blei zur Essigsäure wie $1: 1,3$, daher $3 \mathrm{~Pb}$ auf $4 \mathrm{C}^{2} \mathrm{H}^{3} \mathrm{O}^{2}$; folglich ist auch hier nur $2 / 3$ essigsaures Blei in Lösung. Der Ueberschuss des vorgeschriebenen Bleioxyds ist im Rückstand geblieben und so ist der einzige Unterschied eine unbedeutend grössere Concentration.

Bei dieser Gelegenheit will ich darauf aufmerksam machen, dass die concentrirte Lösung von normalem Bleiacetat, 1 Theil Bleizucker in 2,3-3 Theilen Wasser, Lackmuspapier nicht röthet, wie in den meisten Lehrbüehern angegeben wird, sondern geröthetes Lackmuspapier bläut, also alkalische Reaction besitzt, wie dies, wenn ich nicht irre, zuerst Flückiger (Pharmaceutische Chemie S. 796) beobachtet hat. Wird diese concentrirte Lösung mit mehr Wasser verdünnt, so röthet sie Lackmuspapier und verliert beim Kochen Essigsäure. Hier findet augenscheinlich ein durch das Wasser bewirkter Zerfall in freie Essigsäure und $2 / s$ basisches Salz statt und wahrscheinlich nur auf der Bildung des letzteren beruht die Einwirkung der Kohlensäure durch Fällung des Carbonats, welche bekanntlich um so reichlicher stattfindet, je verdünnter die Lösung des Bleizuckers ist.

Auch hierin tritt unverkennbar die Analogie des $2 / 3$ Bleiacetats mit den $2 / 3$ Acetaten des Aluminiums und Eisens hervor, da diese in Lösung ebenfalls neben freier Essigsäure bestehen.

\section{Nitroglyeerin.}

Der Salpetersäureester des Glycerylalkohols, das Trinitrin oder Nitroglycerin, obwohl ihm dieser letztere Name nicht zukommt, da es keine Nitroverbindung ist, wurde im Jahre 1847 von Sobrero im Laboratorium von Pelouze entdeckt. Er erhielt diese Verbindung durch vorsichtiges Eintropfen von 1/6 Volumen Glycerin in ein unausgesetzt kalt gehaltenes Gemisch von 2 Vol. Schwefelsäure p. sp. 1,840 und 1 Volumen Salpetersäure p. sp. 1,53, Eingiessen der Mischung, sobald sich in ihr eine Oelschicht abschied, in das 15-bis 20fache Volumen Wasser. Das abgeschiedene, schwach gelbliche Oel wurde dann bis zum Verschwinden der sauren Reaction mit 